\title{
The association between chronic exposure to video game violence and affective picture processing: an ERP study
}

\author{
Kira Bailey • Robert West • Craig A. Anderson
}

Published online: 4 April 2011

(C) Psychonomic Society, Inc. 2011

\begin{abstract}
Exposure to video game violence (VGV) is known to result in desensitization to violent material and may alter the processing of positive emotion related to facial expressions. The present study was designed to address three questions: (1) Does the association between VGV and positive emotion extend to stimuli other than faces, (2) is the association between VGV and affective picture processing observed with a single presentation of the stimuli, and (3) is the association between VGV and the response to violent stimuli sensitive to the relevance of emotion for task performance? The data revealed that transient modulations of the event-related potentials (ERPs) related to attentional orienting and sustained modulations of the ERPs related to evaluative processing were sensitive to VGV exposure.
\end{abstract}

\section{Keywords Emotion·erp}

Video games represent one of the most pervasive forms of leisure activity in modern culture, being played in $68 \%$ of American households (Entertainment Software Association, 2010). Eighty-eight percent of individuals 8-18 years of age have reported that they occasionally play video games, and $23 \%$ have reported playing video games at least once per day (Gentile, 2009). Exposure to video game violence (VGV) that involves shooting, stabbing, punching, kicking, and blunt trauma to characters within a game is associated with an increase in aggressive thoughts, feelings, and actions (Anderson, Gentile, \& Buckley, 2007; Anderson,

K. Bailey $(\triangle) \cdot$ R. West $\cdot$ C. A. Anderson

Center for the Study of Violence, Department of Psychology,

Iowa State University,

W112 Lagomarcino Hall,

Ames, IA 50011, USA

e-mail: kmbailey@iastate.edu
Shibuya, Ihori, Swing, Bushman, Sakamoto, \& Saleem, 2010) and a decrease in prosocial or helping behavior (Anderson et al., 2010; Bushman \& Anderson, 2009; Gentile, Anderson, Yukawa, Ihori, Saleem, Ming, \& Sakamoto, 2009). These effects, together with the extensive use of video games beginning in childhood and continuing through adulthood, lead one to wonder what impact this media has on the emotional life of the individual. The present study addressed this question by examining the association between individual differences in exposure to VGV and the neural correlates of affective picture processing, using event-related brain potentials (ERPs).

\section{Video games and desensitization to violence}

One consequence of exposure to $\mathrm{VGV}$ is that individuals may become desensitized to violence (Anderson et al., 2007; Barlett, Anderson, \& Swing, 2009; Bushman \& Anderson, 2009; Carnagey, Anderson, \& Bushman, 2007; Funk, Baldacci, Pasold, \& Baumgardner, 2004). As Carnagey et al. noted, the term desensitization has been used in many different ways in the social, clinical, and media violence domains, a practice that has led to considerable confusion. Theoretically, desensitization can be defined as "a reduction in emotion-related physiological reactivity to real violence" (Carnagey et al., 2007, p. 490). Other common usages of the term usually refer to measures of behavior, attitudes, or self-reported emotions that theoretically result from desensitization, such as reductions in helping behavior or in empathy toward victims of violence or injury.

Several studies have linked VGV to physiological indicators of desensitization. For example, Carnagey et al. (2007) demonstrated that brief exposure to VGV influences 
activation of the autonomic nervous system. In that study, individuals played a violent or a nonviolent video game for $20 \mathrm{~min}$. Immediately after playing the game, individuals viewed a 10-min video clip of real-world violence (e.g., a prison beating) while heart rate and skin conductance were measured. Those who played the violent video game demonstrated reduced cardiac reactivity and a diminished skin conductance response while watching the video, relative to those who played the nonviolent video game. Bushman and Anderson (2009) tested one consequence of physiological desensitization, using the same games. Specifically, these investigators sought to determine whether desensitization was associated with a decrease in the likelihood of aiding a victim of violence in those exposed to VGV. In this study, individuals played one of the video games before a fight was staged outside of a laboratory toward the end of the study. Participants who had played the violent video game were less likely to report hearing the fight, judged the fight as less serious, and were slower to respond when they did offer help than those participants who had played the nonviolent video game (Bushman \& Anderson, 2009).

Bartholow, Bushman, and Sestir (2006) used ERPs to examine the neural basis of desensitization associated with individual differences in chronic exposure to VGV. To assess exposure to VGV, participants listed their top five favorite video games and rated the level of violent content and amount of time spent playing each game. On the basis of these measures, individuals were classified as either violent video gamers or nonviolent video gamers. Then individuals viewed neutral (e.g., man riding a bicycle), negative violent (e.g., man holding a knife to a woman's throat), and negative nonviolent (e.g., decaying dog corpse) pictures from the International Affective Picture System (IAPS; Lang, Bradley, \& Cuthbert, 2005) while ERPs were recorded from the scalp.

The investigators were particularly interested in the association between individual differences in exposure to VGV and the amplitude of the P3 component elicited by violent and negative nonviolent pictures. The amplitude of the P3 was taken as an index of the amount of evaluative processing directed to the content of the pictures, with the expectation that desensitization to violence would result in an attenuation of the amplitude of the P3 for violent pictures in the violent video gamers. Consistent with this prediction, the amplitude of the P3 for violent pictures was attenuated in violent video gamers, relative to nonviolent video gamers. In contrast, the amplitude of the P3 for nonviolent negative pictures was similar for the violent and nonviolent video gamers. The present study builds on the findings of Bartholow et al. (2006) by examining a broader range of ERP components related to early attentional and later evaluative processes in high- and low-violent video gamers.

\section{The influence of VGV on positive affect}

Some evidence indicates that exposure to violent media may alter the experience of positive emotion, as well as desensitizing individuals to violence. This effect was reported in two studies by Kirsh and colleagues (Kirsh \& Mounts, 2007; Kirsh, Mounts, \& Olczak, 2006) that investigated the association between exposure to media and video game violence and the happyface advantage (i.e., the finding that people are typically faster to respond to happy than to angry facial expressions; Billings, Harrison, \& Alden, 1993; Leppanen, Tenhunen, \& Heitanen, 2003) in a change detection task. In the study by Kirsh et al., a neutral face morphed into a happy or angry face, and participants pressed a key when the change was noticed. Individuals low in media violence exposure were faster to identify a neutral face morphing into a happy face than a neutral face morphing into an angry face, demonstrating the happyface advantage. In contrast, individuals high in media violence exposure were faster to identify a neutral face morphing into an angry face than a neutral face morphing into a happy face. Kirsh and Mounts extended this finding in a study where participants played a violent or nonviolent video game for $15 \mathrm{~min}$ before completing the facemorphing task. Individuals who played a violent video game were slower to identify neutral faces morphing to happy than were individuals who had played a nonviolent video game. The findings of these studies demonstrate that both chronic and acute expose to violent media can decrease one's sensitivity to the expression of positive emotion in the face. The present study was designed to determine whether the association between exposure to VGV and the processing of positive information extends to stimuli other than faces.

\section{The neural correlates of affective picture processing}

The literature using ERPs to investigate the neural correlates of affective picture processing has revealed differential neural activity related to the affective valence of pictures beginning as early as $100 \mathrm{~ms}$ after stimulus onset and persisting for the next 1,000-2,000 ms (for reviews, see Codispoti, Ferrari, \& Bradley, 2007; Olofsson, Nordin, Sequeira, \& Polich, 2008). The early posterior negativity (EPN) and late positive potential (LPP) represent two components of the ERPs that are commonly modulated by the valence of pictures. The EPN reflects a transient negativity over the posterior region of the scalp between 200 and $300 \mathrm{~ms}$ after stimulus onset. The EPN distinguishes emotionally valenced pictures from neutral pictures (Codispoti et al., 2007; Schupp, Junghofer, Weike, \& Hamm, 2003) and is greater in amplitude for highly arousing pictures than for less arousing pictures that have the same valence (Schupp et al., 2003). On the basis of these findings, the EPN is thought to reflect the allocation of 
attention to emotionally arousing stimuli (Schupp, Junghofer, Weike, \& Hamm, 2004).

The LPP reflects a sustained positivity, extending from the central to the parietal region of the scalp, that is greater in amplitude for negative and positive pictures than for neutral pictures beginning around $300 \mathrm{~ms}$ after stimulus onset (Carretie, Hinojosa, Albert, \& Mercado, 2006; Hajcak, Dunning, \& Foti, 2009; Ito, Larsen, Smith, \& Cacioppo, 1998; Olofsson et al., 2008) and appears to overlap the P3 component in time. The amplitude of the LPP is greater for affectively valenced pictures than for neutral pictures and is thought to result from a tendency for stimuli that are motivationally or evolutionarily significant to capture attention (Keil et al., 2002; Schupp et al., 2000; Schupp et al., 2004). Some investigators have reported that the amplitude of the LPP is greater for negative pictures than for positive pictures (Ito \& Cacioppo, 2005; Ito et al., 1998), a finding that is thought to reflect the negativity bias that has been described in various domains of social cognition (Baumeister, Bratslavsky, Finkenauer, \& Vohs, 2001). Recent work using temporal-spatial principal components analysis (PCA) has revealed that the LPP reflects the activity of multiple components of the ERPs whose distribution extends from the central to the occipital-parietal regions of the scalp between 400 and 2,000 ms after stimulus onset (Foti, Hajcak, $\&$ Dien, 2009). Some of these components reveal similar neural activity for positive and negative pictures, reflecting a general sensitivity to affective information. In contrast, other components reveal a stronger response to negative than to positive pictures, possibly reflecting the expression of the negativity bias.

\section{The present study}

The present investigation was designed to extend the findings of previous research by examining the association between individual differences in chronic VGV exposure and the neural correlates of affective picture processing. In two experiments, participants viewed positive, negative nonviolent, negative violent, and neutral pictures from the IAPS (Lang et al., 2005). In Experiment 1, participants rated the pictures for colorfulness; in Experiment 2, participants rated the pictures for colorfulness, pleasantness, and how threatening the pictures were. Positive pictures were included to determine whether the findings of Kirsh et al. (2006) using faces would extend to scenes eliciting positive emotion. Varying the rating task across blocks of trials in Experiment 2 allowed us to determine whether the association between individual differences in VGV exposure and affective picture processing observed in Experiment 1 was sensitive to the degree that the emotional content of the pictures was relevant to task performance. This approach can be contrasted with that of Bartholow et al. (2006), where individuals performed a single orienting task that did not require an overt response. This element of the design makes it impossible to know whether violent and nonviolent video gamers engaged in similar processing during picture viewing.

Work demonstrating that the LPP reflects the activity of multiple components of the ERPs that are differentially sensitive to various aspects of emotion (Foti et al., 2009) highlights the need to incorporate a statistical technique that allows one to integrate information related to time course, topography, and task conditions in a single analysis. To address this issue in the present study, partial least squares (PLS) analysis was used to analyze the ERP data (Lobaugh, West, \& McIntosh, 2001; McIntosh \& Lobaugh, 2004). PLS analysis is a multivariate statistical technique that is similar to PCA (Gorsuch, 1983) and allows one to decompose the full time course and topography of the scalp-recorded ERPs into a set of orthogonal latent variables that capture differences in mean amplitude between task conditions across time and space. For instance, in a recent study using ERPs in combination with PLS analysis and the picture-rating task incorporated in the present experiments, we observed a pair of significant latent variables (Bailey, West, \& Olson, 2011). One latent variable appeared to capture the expression of the negativity bias, reflecting a contrast of negative and violent pictures with positive pictures of people and erotic pictures. The second latent variable appeared to capture the neural correlates of processing erotic pictures,since it contrasted erotic pictures with neutral pictures and other positive pictures. These findings indicate that PLS analysis can be used to identify patterns of neural activity that are differentially sensitive to various aspects of affective picture processing.

\section{Experiment 1}

In Experiment 1, individuals high or low in chronic exposure to VGV rated the colorfulness of neutral, positive, negative nonviolent, and negative violent pictures. In the study by Bartholow et al. (2006), each of the pictures was presented 15 times, making it impossible to determine whether the reduction in the amplitude of the LPP elicited by violent pictures resulted from a general reduction in the sensitivity to these materials or from faster habituation within the experiment to these stimuli. To address this issue, each picture was presented only once in the present experiment. On the basis of the findings of Bartholow et al., the amplitude of the LPP for violent pictures was expected to be attenuated in high gamers, relative to low gamers, and the amplitude of the LPP for negative nonviolent pictures was expected to be similar for high and low gamers. In the PLS analysis, this association could be expressed on the latent variable capturing the negativity bias, reflecting a contrast 
between negative violent and negative nonviolent pictures, and positive and neutral pictures. In low gamers, the magnitude of the brain scores should be similar for negative violent and negative nonviolent pictures, and in high gamers, the magnitude of the brain scores for negative violent pictures should be reduced relative to the negative nonviolent pictures. If the findings of Kirsh et al. (2006) extend to positive stimuli other than faces, the amplitude of the ERPs that are sensitive to processing positive pictures should be attenuated in high gamers, relative to low gamers. In the PLS analysis, this association could be expressed on a latent variable that contrasts positive pictures with neutral pictures, and the magnitude of this contrast should be reduced in high gamers, relative to low gamers.

\section{Method}

\section{Participants}

Forty-two males from Iowa State University participated in this experiment. Participants were recruited on the basis of their responses to a media usage questionnaire that they completed twice:once at least 2 weeks prior to participation in the laboratory session, as part of a larger screening exercise, and once at the end of the laboratory session. On the basis of responses from the laboratory session, the sample included 21 high gamers $(M=33 \mathrm{~h}$ per week, range $=9-64 \mathrm{~h}$ per week $)$ and 21 low gamers $(M=3 \mathrm{~h}$ per week, range $=0-9 \mathrm{~h}$ per week). The high gamers $(M=2.23, S D=0.64)$ reported greater familiarity with a set of four popular violent video games than did the low gamers $(M=1.26, S D=0.31), t(39)=$ $2.71, p<.01$. The high gamers $(M=19.2, S D=1.75)$ and low gamers $(M=20.71, S D=3.18)$ were similar in years of age and in their distribution of handedness (high gamers, right $=17$, ambidextrous $=1$, left $=3$; low gamers, right $=17$, ambidextrous $=3$; Oldfield, 1971) .

\section{Materials}

Media usage questionnaire This questionnaire included three higher order questions. Two questions asked the individual to indicate the number of hours spent playing video games on a typical weekday (Question 1, Monday through Friday) or weekend (Question 2, Saturday and Sunday) for each of four time periods ( 6 a.m. to noon, noon to 6 p.m., 6 p.m. to midnight, and midnight to 6 a.m.). The third question asked the participant to indicate how often he played any version of each of four popular video games (i.e., Counterstrike, Grand Theft Auto, Resident Evil, and Unreal Tournament) on a 5-point scale (1, indicating have never played, to 5 , indicating play very often). Participants who reported playing 10 or more hours of any video games per week and indicated greater experience (i.e., responded with a 3,4 , or 5 on the scale) with the four video games in the screening session were recruited as high gamers. Participants who reported playing less than $5 \mathrm{~h}$ of any video games per week and indicated little experience (i.e., responded with a 1 or 2 on the scale) with the four video games in the screening session were recruited as low gamers. The internal reliability was high for the number of hours played (screening coefficient $\alpha=.85$, laboratory session coefficient $\alpha=.83$ ), and the test-retest reliability was acceptable $(r=.76)$. The internal reliability was acceptable for the level of action game exposure (screening coefficient $\alpha=.71$, laboratory session coefficient $\alpha=.72$ ), as was the test-retest reliability of this measure $(r=.77)$.

Affect measures The Behavior Inhibition/Behavior Activation Scales (BIS/BAS; Carver \& White, 1994) and the Beck Depression Inventory (BDI; Beck, Ward, Mendelson, Mock, \& Erbaugh, 1961) were administered to measure individual differences in trait affect. The BIS/BAS scale contained 19 statements that were rated on a 4-point scale with 1 being strong agreement and 4 being strong disagreement with the statement. On the BDI, participants selected which of 4 statements in each of 19 groups of statements best described how he had felt over the last 2 weeks. Items related to thoughts of suicide and loss of interest in sex were omitted from the BDI.

Picture-rating task The stimuli were pictures from the IAPS (Lang et al., 2005). Valence ratings from the normative dataset were used to divide the pictures into those representing negative, neutral, and positive emotion. The pictures were further divided so that negative $(M=6.2, S D=0.57$, range = 5.04-7.29) and positive $(M=5.9, S D=0.71$, range $=3.55$ 7.35) pictures would have overlapping arousal ratings. One hundred twenty pictures were selected for the task and included 40 neutral, 40 positive, and 40 negative pictures; the negative picture were further divided into 20 violent $(M=$ $6.3, S D=0.52)$ and 20 nonviolent $(M=6.1, S D=0.62)$ pictures with similar arousal ratings, $t(38)=0.93, p=.36{ }^{1}$ All the pictures contained people. Neutral pictures contained

\footnotetext{
${ }^{1}$ Neutral (IAPS numbers: 2102, 2104, 2191, 2215, 2221, 2235, 2305, 2383, 2393, 2396, 2397, 2410, 2440, 2480, 2485, 2487, 2499, 2513, $2514,2516,2518,2579,2580,2593,2595,2597,2620,2635,2745.1$, 2830, 2840, 2850, 2870, 4605, 7493, 7496, 7506, 8010, 8311, 8465). Positive (IAPS numbers: 2058, 2071, 2160, 2209, 2216, 2345, 2346, 4599, 4626, 4640, 4641, 5460, 5470, 5621, 5623, 5629, 5833, 7325, $7502,8030,8034,8080,8090,8180,8185,8186,8190,8200,8210$, $8300,8350,8370,8380,8400,8420,8470,8490,8496,8499,8540)$. Negative violent (IAPS numbers: 2683, 3500, 3530, 6212, 6250, $6312,6313,6315,6350,6360,6530,6540,6550,6560,6571,6821$, $9423,9424,9427,9428)$. Negative nonviolent (IAPS numbers: 2710, 2730, 2751, 3005.1, 3168, 3170, 3230, 3261, 3266, 6834, 8485, 9050, 9410, 9421, 9635.1, 9800, 9810, 9903, 9910, 9925).
} 
images of people with neutral expressions or groups of people in public places. Positive pictures contained children, families, and athletes. Erotic pictures were not included, in order to make more direct comparisons with the findings of Bartholow et al. (2006). Violent pictures were defined as those that included at least two people where one person either held a weapon or was attacking the other person (e.g., a man choking a woman). Nonviolent pictures included images of grief, loss, and accidents and did not include mutilations. The label "negative" is used to refer to the negative nonviolent pictures, and the label "violent" is used to refer to the negative violent pictures. Pictures were $512 \times 384$ pixels and were presented on a white background.

\section{Procedure}

All stimuli were presented using E-Prime 1.2 software (Psychology Software Tools, Pittsburgh, PA). Pictures were displayed on the screen until the participant responded, followed by a blank screen for $500 \mathrm{~ms}$. Participants were instructed to rate how colorful he found each picture, using the keys "v,"“b," "n," and "m," with "v" labeled as least colorfuland " $\mathrm{m}$ " labeled as most colorful. After instructions had been given, participants were shown 3 practice pictures, 1 picture from each valence category, that were not included in the 120 pictures, to practice the rating task. The pictures were presented in a different random order for each participant.

The fitting of the electro-cap was briefly described to the participant when he arrived for the study. The participant then gave informed consent and completed the Edinburgh Handedness Inventory (Oldfield, 1971), the BIS/BAS scales (Carver \& White, 1994), and the BDI (Beck et al., 1961). After the cap had been fitted, participants were moved to the testing room and were asked to sit comfortably in front of the computer monitor. Participants were asked to limit eye and head movements during recording. Following testing, participants completed the media usage questionnaire.

\section{Electrophysiological recording and analysis}

The electroencephalogram (EEG;bandpass, 0.02-150 Hz; digitized at $500 \mathrm{~Hz}$; gain, 1,000; 16-bit A/D conversion) was recorded from an array of 68 tin electrodes on the basis of a modified 10-10 system using an electro-cap (ElectroCap International, Eaton, $\mathrm{OH})$. Vertical and horizontal eye movements were recorded from electrodes placed beside and below the right and left eyes. During recording, all electrodes were referenced to electrode $\mathrm{Cz}$, then rereferenced to an average reference for data analysis. A $0.1-$ to $8-\mathrm{Hz}$ zero-phase shift filter was applied before averaging.
Ocular artifacts associated with blinks and saccades were removed from the data, using a covariance-based technique including empirically derived estimates of the EEG associated with artifact and artifact-free data (Electromagnetic Source Estimation; Source-Signal Imaging, San Diego). ERP analysis epochs were obtained offline with $-200 \mathrm{~ms}$ of prestimulus activity and 1,000 ms of poststimulus activity for each picture type.

\section{Partial least squares (PLS) analysis}

PLS analysis was applied to an ERP data matrix representing the data for participants and conditions in the rows, and the amplitudes for time points between 0 and 1,000 $\mathrm{ms}$ at 64 channels (excluding the four ocular electrodes) in the columns. The input (deviation) matrix for the PLS analysis was obtained by mean-centering the columns of the data matrix with respect to the grand mean. Singular value decomposition (SVD) was performed on the deviation matrix to identify the structure of the latent variables. Eight latent variables were extracted from the deviation matrix, with the first six capturing mean differences in amplitude across groups and/or conditions and the seventh and eighth capturing the grand mean for the low and the high gamers, respectively. Three outputs were obtained from the SVD that were used to interpret the relationships between ERP amplitude, task design, and gaming status. The first was a vector of singular values that are similar to eigenvalues and represent the unweighted magnitude of each latent variable. The singular values were used to calculate the percentage of task-related variance attributable to each latent variable. The second and third outputs represent the structure of the latent variables and are orthogonal pairs of vectors that are similar to component loadings in PCA. One vector defines the contrasts among conditions scaled for amplitude (brain scores), and the other vector represents the electrode saliences that reflect the spatial-temporal distribution of the latent variable across the scalp. The electrode saliences reflect components or modulations of the ERP waveforms that differ in amplitude across task conditions (e.g., an effect on the P3 might reflect stable saliences over the parietal region of the scalp between 400 and $600 \mathrm{~ms}$ ).

The significance of the latent variables' singular values was determined using a permutation test (500 replications) that provided the exact probability of observing the latent variable singular value by chance (e.g., $p=.01$ ); the stability of the electrode saliences at each time point and location on the scalp and the brain scores for the task conditions were established through bootstrap resampling (500 replications), which provided a standard error for each of the electrode saliences and brain scores. The ratio of the salience or brain score to its bootstrapped standard error was approximately equivalent to a $\mathrm{z}$-score; therefore, 
bootstrap ratios greater than 3.0 could be taken to indicate saliences that differed from zero at the $p<.001$ level. MATLAB code to perform the PLS analyses can be obtained at http://www.rotman-baycrest.on.ca.

\section{Results}

\section{Affect measures}

To examine the association between trait affect and video game experience, the scores of the low and high gamers on the BIS/BAS scales and the BDI were compared. Low gamers $(M=19, S D=3)$ and high gamers $(M=18, S D=3)$ reported similar scores on the BIS scale, $t(40)=-1.44, p=$ .16. Low gamers $(M=40, S D=4)$ and high gamers $(M=$ $37, S D=5$ ) were not significantly different on the BAS scale, $t(40)=-1.85, p=.07$. There was no difference between the low gamers $(M=7, S D=7)$ and high gamers $(M=7, S D=5)$ on the BDI, $t(40)=0.45, p=.66$. These data indicate that the levels of trait affect were similar for the two groups.

\section{Picture-rating data}

Mean ratings of colorfulness (Table 1) were analyzed in a 2 (gamer status: low, high) $\times 4$ (picture type: neutral, negative, violent, positive) ANOVA. The main effect of picture type was significant, $F(3,117)=48.99, p=.001$, $\eta_{\mathrm{p}}{ }^{2}=.55$. This reflected significant differences in the colorfulness ratings of all picture types except negative and violent pictures. The main effect of gamer status and the gamer status $\times$ picture type interaction were not significant, $F_{\mathrm{s}}<1.56, p \mathrm{~s}>.64, \eta_{\mathrm{p}}{ }^{2}=.04$.

Table 1 Mean pictures ratings for Experiments 1 and 2

\begin{tabular}{|c|c|c|c|c|c|}
\hline \multicolumn{6}{|c|}{ Experiment 1} \\
\hline & & Neutral & Negative & Violent & Positive \\
\hline \multirow[t]{2}{*}{ Color } & $M$ & $2.56_{a, b, c}$ & $2.31_{\mathrm{e}}$ & $2.37_{\mathrm{f}}$ & 2.95 \\
\hline & $S D$ & 0.23 & 0.33 & 0.37 & 0.30 \\
\hline \multicolumn{6}{|c|}{ Experiment 2} \\
\hline & & Neutral & Negative & Violent & Positive \\
\hline \multirow[t]{2}{*}{ Color } & $M$ & $2.65_{\mathrm{b}, \mathrm{c}}$ & $2.20_{\mathrm{d}, \mathrm{e}}$ & $2.35_{\mathrm{f}}$ & 3.03 \\
\hline & $S D$ & 0.25 & 0.38 & 0.41 & 0.32 \\
\hline \multirow[t]{2}{*}{ Pleasant } & $M$ & $2.80_{\mathrm{a}, \mathrm{b}, \mathrm{c}}$ & $1.26_{\mathrm{e}}$ & $1.22_{\mathrm{f}}$ & 3.49 \\
\hline & $S D$ & 0.49 & 0.29 & 0.30 & 0.48 \\
\hline \multirow[t]{2}{*}{ Threat } & $M$ & $1.28_{\mathrm{a}, \mathrm{b}, \mathrm{c}}$ & $3.22_{\mathrm{d}, \mathrm{e}}$ & $3.52_{\mathrm{f}}$ & 1.44 \\
\hline & $S D$ & 0.28 & 0.58 & 0.40 & 0.41 \\
\hline
\end{tabular}

Posthoc paired contrasts using a Bonferroni adjustment of $p=.008$.(a) Neutral vs. negative.(b) Neutral vs. violent.(c) Neutral vs. positive.(d) Negative vs. violent.(e) Negative vs. positive.(f) Violent vs. positive

\section{ERP data}

The grand-averaged ERPs at select electrodes for the high and low gamers are presented in Figs. 1 and 2. These data reveal a small EPN over the occipital-parietal region at around $250 \mathrm{~ms}$ after stimulus onset that appears to distinguish negative pictures from the other pictures in low gamers and negative and violent pictures from neutral pictures in the high gamers (Fig. 2). Beginning at around $500 \mathrm{~ms}$ after stimulus onset, the LPP can be seen in both groups at the parietal and central-parietal electrodes. There was also slow wave activity over the frontal region of the scalp that distinguished the four types of pictures from one another.
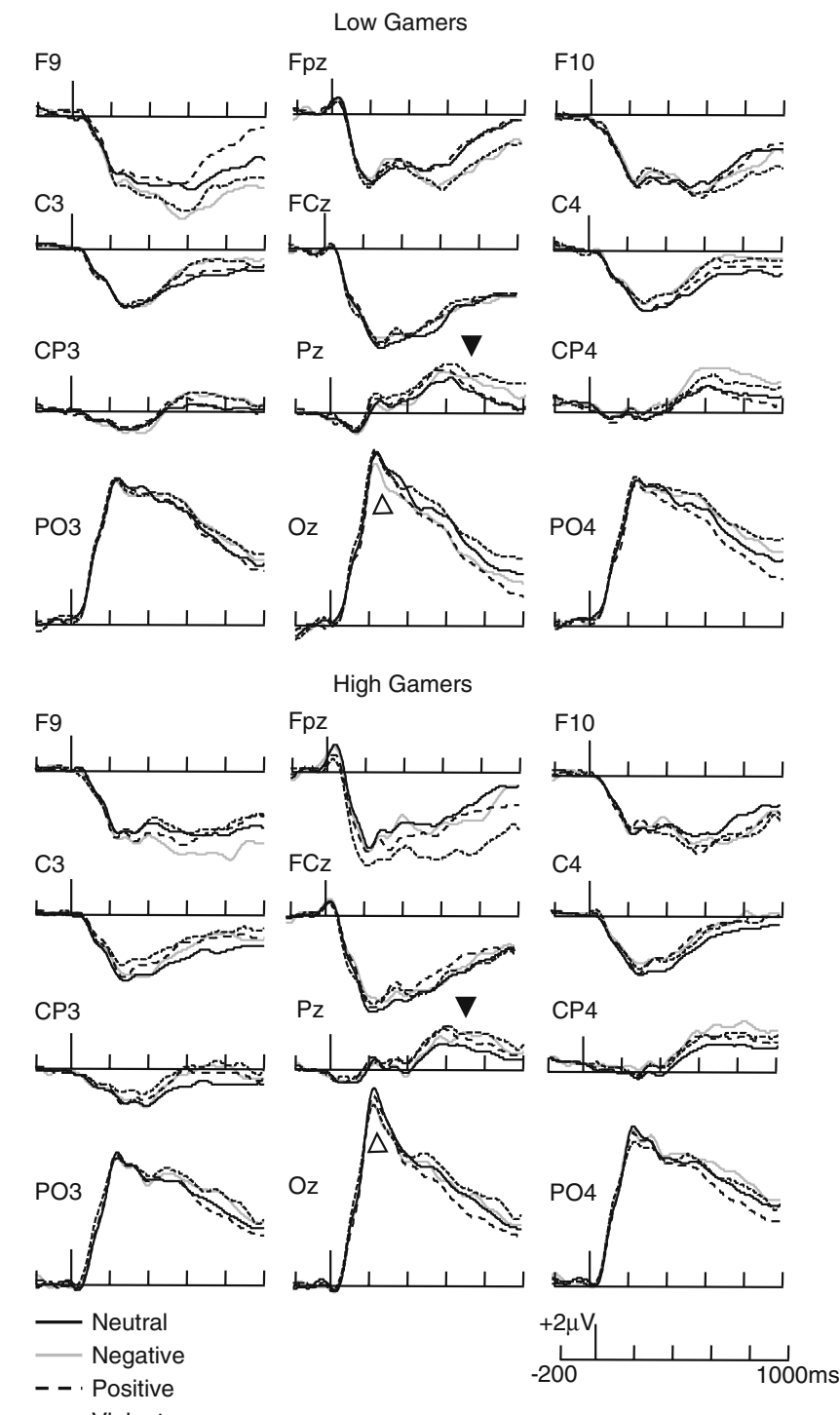

---- Violent

Fig. 1 Grand-averaged ERPs at select electrodes for Experiment 1, demonstrating the time course of the EPN (open arrows) and LPP (filled arrows) in the high and low gamers. The taller bar marks the onset of the stimulus, while the shorter bars represent 200-ms increments, and positive is plotted up 


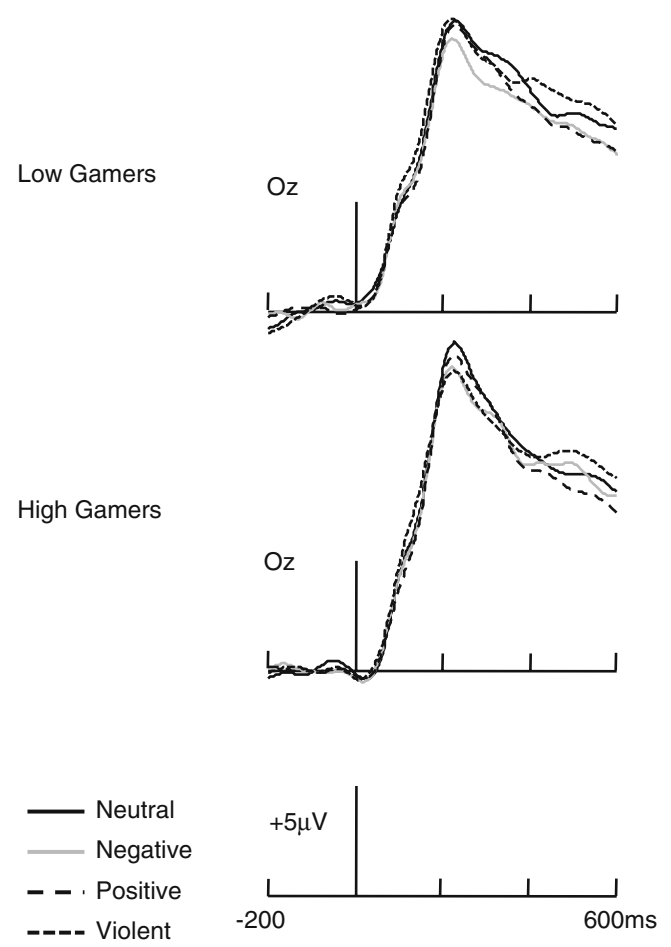

Fig. 2 Grand-averaged ERPs at electrode Oz, demonstrating the EPN. The taller bar marks the onset of the stimulus, while the shorter bars represent 200-ms increments, and positive is plotted up

The permutation test for the PLS analysis revealed two significant latent variables $(p=.01, p=.04)$ that accounted for $48.49 \%$ and $22.17 \%$ of the cross-block covariance, respectively. The first latent variable represented a contrast of negative and violent pictures with neutral and positive pictures (Fig. 3, top panel). The magnitude of the brain scores was similar for negative pictures for the high and low gamers, while the magnitude of the brain scores for the violent pictures was reduced in the high gamers relative to the low gamers. These findings are consistent with those of Bartholow et al. (2006) and may reveal desensitization to violence in the high gamers. The electrode saliences revealed three stable time periods of neural activity. The first reflected a transient negativity over the midline frontal region around $125 \mathrm{~ms}$ after stimulus onset (electrode $\mathrm{FCz}$ ). The second reflected a transient negativity over the occipital region around $280 \mathrm{~ms}$ after stimulus onset, likely reflecting the EPN (electrode Oz). The third reflected a sustained positivity over the parietal region and a negativity over the left lateral frontal region between 500 and 1,000 ms after stimulus onset, likely reflecting the LPP (electrodes PO4, F9).

The pattern of brain scores for the second latent variable differed in the low and high gamers. For the low gamers, this latent variable represented a contrast between positive pictures and neutral pictures (Fig. 3, bottom panel). In contrast, for the high gamers, this latent variable contrasted positive and violent pictures with neutral pictures. The electrode saliences revealed two stable time periods of neural activity. The first reflected a transient modulation over the frontal-polar region around $100 \mathrm{~ms}$ after stimulus onset (electrode Fpz), and the second reflected a sustained positivity over the centralparietal region and negativity over the occipital region between 400 and $900 \mathrm{~ms}$ (electrodes C3, CP3, Iz).

\section{Discussion}

The behavioral data revealed that chronic VGV exposure was not associated with individual differences in trait emotion or colorfulness ratings in the picture-rating task. These results of the PLS analysis reveal a number of interesting findings related to the association between chronic VGV exposure and the neural correlates of affective picture processing. The pattern of brain scores for the first latent variable reveals that desensitization to violence can be observed with a single presentation of the stimuli (i.e., the brain score for violent pictures was reduced in the high gamers, relative to the low gamers). These data provide a conceptual replication of the findings of Bartholow et al. (2006), indicating that the previous findings likely do not reflect faster within-task habituation to the violent pictures. The second latent variable appeared to capture the neural correlates of processing positive pictures in the low gamers. Additionally, the magnitude of the contrast between the positive and neutral pictures in the brain scores was similar in the two groups or greater for high gamers. This result is inconsistent with the findings of Kirsh et al. (2006) and may indicate that the association between chronic VGV exposure and positive emotion does not extend to stimuli other than faces. Another interesting finding related to the second latent variable was the clustering of positive and violent pictures in the high gamers, but not the low gamers. Within the general aggression model (Anderson \& Bushman, 2002), this association could reflect the high gamers' extensive history of being rewarded for engaging in violence during game play, resulting in positive and negative stimuli taking on similar hedonic value. However, the novel and unexpected nature of this association suggests that it should be replicated. Finally, the pattern of electrode saliences for both latent variables reveals that chronic VGV exposure is associated with the modulation of a broadly distributed set of neural generators that support relatively transient attentional processing of valence or arousal (i.e., the EPN) and more sustained evaluative processes (i.e., the LPP).

\section{Experiment 2}

Experiment 2 was designed to replicate and extend the findings of Experiment 1. In this experiment, high and low gamers rated the four types of pictures for colorfulness, pleasantness, or level 
Fig. 3 Results of the PLS analysis for Experiment 1. The top panel portrays the brain scores (left) and electrode saliences (right) for the first latent variable. The bottom panel portrays the brain scores and electrode saliences for the second latent variable. The error bars for the brain scores represent the $95 \%$ confidence intervals derived from the bootstrap distribution
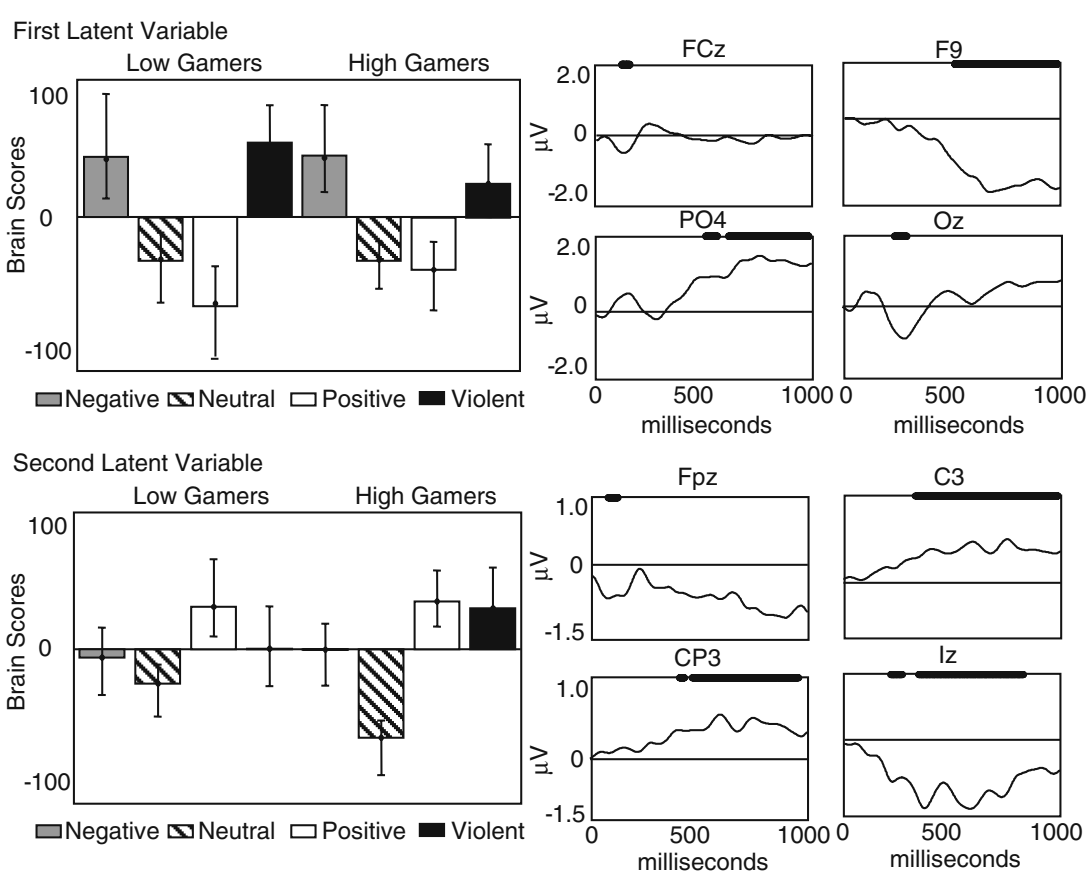

of threat in separate blocks of trials. Varying the orienting task across blocks of trials allowed us to determine whether the association between VGV exposure and the neural correlates of affective picture processing observed in Experiment 1 was relatively automatic or was moderated by the degree that the emotional content of the pictures was relevant to task performance. If the association between $\mathrm{VGV}$ and affective picture processing is relatively automatic (Codispoti et al., 2007), differences in neural activity between high and low gamers should be similar across the three orienting tasks (i.e., the magnitude of the brain scores in the PLS analyses for violent pictures should be reduced in high gamers, relative to low gamers, across all three tasks). In contrast, if the association between $\mathrm{VGV}$ and affective picture processing is sensitive to variation in motivational or attentional factors (Schupp et al., 2004), differences between the high and low gamers may vary with the demands of the orienting tasks (e.g., the magnitude of the brain scores for violent pictures could be increased in high gamers, relative to low gamers, for the threat-rating task, given their experience in detecting threatening stimuli during game play). Additionally, we examined whether the association between VGV and affective picture processing might be related to individual differences in state emotion at the time of testing.

\section{Method}

Participants

Forty-eight males from Iowa State University participated in this experiment. The recruitment procedure was identical to that in Experiment 1. The sample included 24 high gamers ( $M=26 \mathrm{~h}$ per week, range $=9-58 \mathrm{~h}$ per week at the time of the laboratory session) and 24 low gamers $(M=4 \mathrm{~h}$ per week, range $=0-14 \mathrm{~h}$ per week at the time of the laboratory session). The high gamers $(M=2.50, S D=0.79)$ reported greater familiarity with the four violent video games than did the low gamers $(M=1.24, S D=0.32), t(46)=2.69, p<$ .01 . The high gamers $(M=19.87, S D=3.52)$ and low gamers $(M=19.73, S D=1.28)$ were similar in years of age and in their distribution of handedness (high gamers, right $=20$, ambidextrous $=3$, left $=1$; low gamers, right $=$ 18 , ambidextrous $=4$, left $=2$; Oldfield, 1971).

\section{Materials}

Media usage questionnaire The same questionnaire as that used in Experiment 1 was administered during a screening session and at the end of the laboratory session. The internal reliability was good for the number of hours played (screening coefficient $\alpha=.86$, laboratory session coefficient $\alpha=.83$ ), and the test-retest reliability of this measure was acceptable $(r=.75)$. The internal reliability was acceptable for the level of violent video game exposure (screening coefficient $\alpha=.70$, laboratory session coefficient $\alpha=.71$ ), as was the test-retest reliability of this measure $(r=.80)$.

Emotion scale The emotion scale required participants to rate how strongly each of eight adjectives (bored, sad, energetic, amused, calm, angry, happy, and anxious) described his current emotional state by placing a mark on a $10-\mathrm{cm}$ line that was bounded by the labels "not at all" to "extremely." 
Picture-rating task The stimuli were identical to those used in Experiment 1. Each of the 120 pictures was presented once in three different blocks of trials, so that the participants saw and rated each picture on three dimensions. In separate blocks, participants rated the pictures for pleasantness, level of threat, or colorfulness.

\section{Procedure}

Instructions were presented at the beginning of each block indicating how the participants were to rate the pictures for that block (e.g., "Rate how threatening each picture is on a scale from 1 (least) to 4 (most)"), using the keys "v,"“b,"“n," and "m," with "v" being least and "m" being most. After instructions were delivered, participants were shown three practice pictures, not included in the pictures used for the experimental block. Pictures were presented in a different random order for each participant within a block, and the order of the blocks was counterbalanced across participants. Participants completed the emotion scale prior to beginning the picture-rating task. The procedure was otherwise identical to that in Experiment 1.

\section{Electrophysiological recording and analysis}

The EEG recording was identical to that in Experiment 1. ERP analysis epochs were obtained offline and included $200 \mathrm{~ms}$ of prestimulus activity and $1,000 \mathrm{~ms}$ of poststimulus activity. Averages were obtained for each of the picture types separately for each rating task. The PLS analyses were identical to those in Experiment 1, with a separate analysis performed for each of the rating tasks.

\section{Results}

State emotion

The emotion scale was used to assess current emotional state. A MANOVA with rating of the eight adjectives as dependent measures (Table 2) revealed that the main effect of group was not significant, $F<1$. This indicates that the groups were similar in their emotional state when they began the picture-rating task.

Picture-rating data

Mean ratings of the pictures for the three tasks (Table 1) were analyzed in a set of 2 (gamer status: low, high) $\times 4$ (picture type: neutral, negative, violent, positive) ANOVAs. In the analysis of the colorfulness-rating task, the main effect of picture type was significant, $F(3,138)=78.31, p=$
Table 2 Mean ratings on the emotion scale in Experiment 2

\begin{tabular}{llcc}
\hline & & Low Gamers & High Gamers \\
\hline Bored & $M$ & 27.83 & 33.54 \\
& $S D$ & 20.22 & 22.75 \\
Sad & $M$ & 5.63 & 5.29 \\
& $S D$ & 9.05 & 9.29 \\
Energetic & $M$ & 38.05 & 28.54 \\
& $S D$ & 15.87 & 18.47 \\
Amused & $M$ & 56.96 & 49.29 \\
& $S D$ & 19.46 & 17.89 \\
Calm & $M$ & 66.25 & 68.29 \\
& $S D$ & 12.25 & 12.18 \\
Angry & $M$ & 2.88 & 5.21 \\
& $S D$ & 4.33 & 11.53 \\
Happy & $M$ & 54.21 & 53.92 \\
& $S D$ & 10.59 & 20.24 \\
Anxious & $M$ & 32.71 & 41.04 \\
& $S D$ & 23.25 & 22.57 \\
\hline
\end{tabular}

$.001, \eta_{\mathrm{p}}{ }^{2}=.63$. This reflected significant differences in the colorfulness ratings of all picture types except the neutral and negative pictures (Table 1 ). The gamer status $\times$ picture type interaction was significant, $F(3,138)=5.01, p=.01$, $\eta_{\mathrm{p}}{ }^{2}=.10$, and reflected higher colorfulness ratings for violent pictures by the high gamers $(M=2.47, S D=0.29)$ than by the low gamers $(M=2.23, S D=0.48), t(46)=2.08$, $p=.04$, and no differences between the two groups for the other picture types.

In the analysis of the pleasantness-rating task, the main effect of picture type was significant, $F(3,138)=405.55, p=$ $.001, \eta_{\mathrm{p}}{ }^{2}=.90$. This effect reflected significant differences in the pleasantness ratings of all picture types except negative and violent pictures (Table 1). The main effect of gamer status and the gamer status $\times$ picture type interaction were not significant, $F_{\mathrm{S}}<1, \eta_{\mathrm{p}}{ }^{2}<.03$.

In the analysis of the threat-rating task, the main effect of picture type was significant, $F(3,138)=573.16, p=.001$, $\eta_{\mathrm{p}}{ }^{2}=.93$. This reflected significant differences in the threat ratings of all picture types, with the mean ratings increasing from neutral to positive, from positive to negative, and from negative to violent pictures (Table 1).The main effect of gamer status and the gamer status $\times$ picture type interaction were not significant, $F \mathrm{~s}<1, \eta_{\mathrm{p}}{ }^{2}<.02$.

\section{ERP data}

The grand-averaged ERP data for the four types of pictures and the three rating tasks at four midline electrodes for the high and low gamers are presented in Fig. 4. Consistent with the results of Experiment 1, one can see a small EPN over the occipital-parietal region around $250 \mathrm{~ms}$ after 


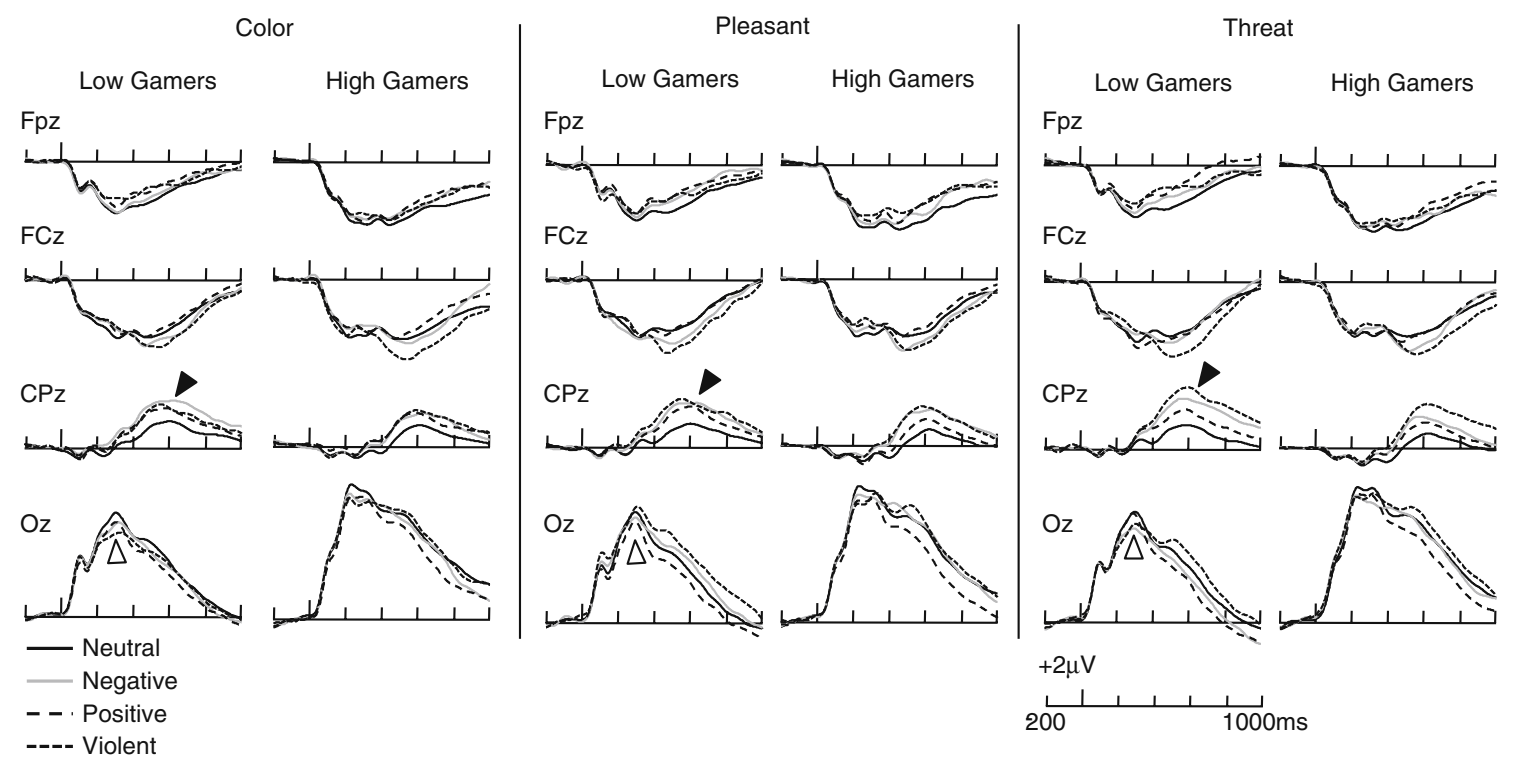

Fig. 4 Grand-averaged ERPs at select electrodes for Experiment 2, demonstrating the time course of the EPN (open arrows) and LPP (black arrows) in high and low gamers across the three tasks. The

stimulus onset, the LPP beginning around $400 \mathrm{~ms}$ after stimulus onset, and slow wave activity over the frontal region of the scalp distinguishing the four types of pictures.

Color-rating task The permutation test revealed three significant latent variables $(p=.001, p=.001, p=.002)$ that accounted for $44.59 \%, 24.99 \%$, and $18.93 \%$ of the crossblock covariance, respectively. The first latent variable represented a contrast of negative and violent pictures with neutral and positive pictures (Fig. 5, top panel), appearing to capture the negativity bias. Unlike in Experiment 1, the magnitude of the brain score for violent pictures was not smaller in the high gamers than in the low gamers. The electrode saliences for the first latent variable revealed two stable time periods of neural activity. The first reflected a transient effect over the occipital region that peaked around $280 \mathrm{~ms}$ after stimulus onset and may reflect the EPN (electrode $\mathrm{Oz}$ ). The second reflected a sustained modulation over the parietal and lateral frontal regions between 400 and $800 \mathrm{~ms}$ after stimulus onset, reflecting the LPP (electrodes $\mathrm{Pz}, \mathrm{F} 10)$.

The second latent variable represented a contrast between positive and neutral pictures in the high gamers and between positive and neutral and negative pictures in the low gamers (Fig. 5, middle panel). This latent variable may capture the neural correlates of processing positive affective information. The electrode saliences revealed two time periods of stable neural activity. The first reflected a transient modulation over the parietal-occipital region that peaked around $150 \mathrm{~ms}$ after stimulus onset (electrode PO4). The second reflected a sustained modulation over the taller bar marks the onset of the stimulus, while the shorter bars represent 200-ms increments, and positive is plotted up

occipital and right lateral frontal regions between 400 and $800 \mathrm{~ms}$ after stimulus onset (electrode F3, PO4). As was the case for the second latent variable in Experiment 1, the pattern of brain scores for this latent variable seems inconsistent with the idea that there is an association between VGV exposure and positive affect.

The third latent variable represented a contrast between negative and violent pictures, with this difference appearing to be stronger in low gamers than in high gamers (Fig. 5, bottom panel). The electrode saliences revealed one stable time period of neural activity that reflected a negativity extending from the midline central-parietal to the frontalcentral region over the left hemisphere (electrodes FC5, $\mathrm{CP} 1$ ) and a positivity extending from the parietal to the lateral frontal region over the right hemisphere between 300 and $700 \mathrm{~ms}$ after stimulus onset (electrode FT10).

Pleasantness-rating task The permutation test revealed two significant latent variables $(p=.001, p=.001)$ that accounted for $65.02 \%$ and $22.23 \%$ of the cross-block covariance, respectively. The first latent variable represented a contrast between the negative and violent pictures and the neutral and positive pictures (Fig. 6, top panel), appearing to capture the negativity bias. The difference in the magnitude of the brain scores for negative and violent pictures was smaller in high gamers than in low gamers, possibly indicative of desensitization to violence in the high gamers. The electrode saliences revealed one period of stable neural activity for this latent variable, reflecting a sustained parietal positivity and lateral frontal negativity between 400 and 1,000 ms after stimulus onset, reflecting the LPP (electrodes Pz, F9, F10). 
Fig. 5 Results of the PLS analysis for the color-rating task in Experiment 2. The top panel portrays the brain scores (left) and electrode saliences (right) for the first latent variable. The middle panel portrays the brain scores and electrode saliences for the second latent variable. The bottom panel portrays the brain scores and electrode saliences for the third latent variable. The error bars for the brain scores represent the $95 \%$ confidence intervals derived from the bootstrap distribution
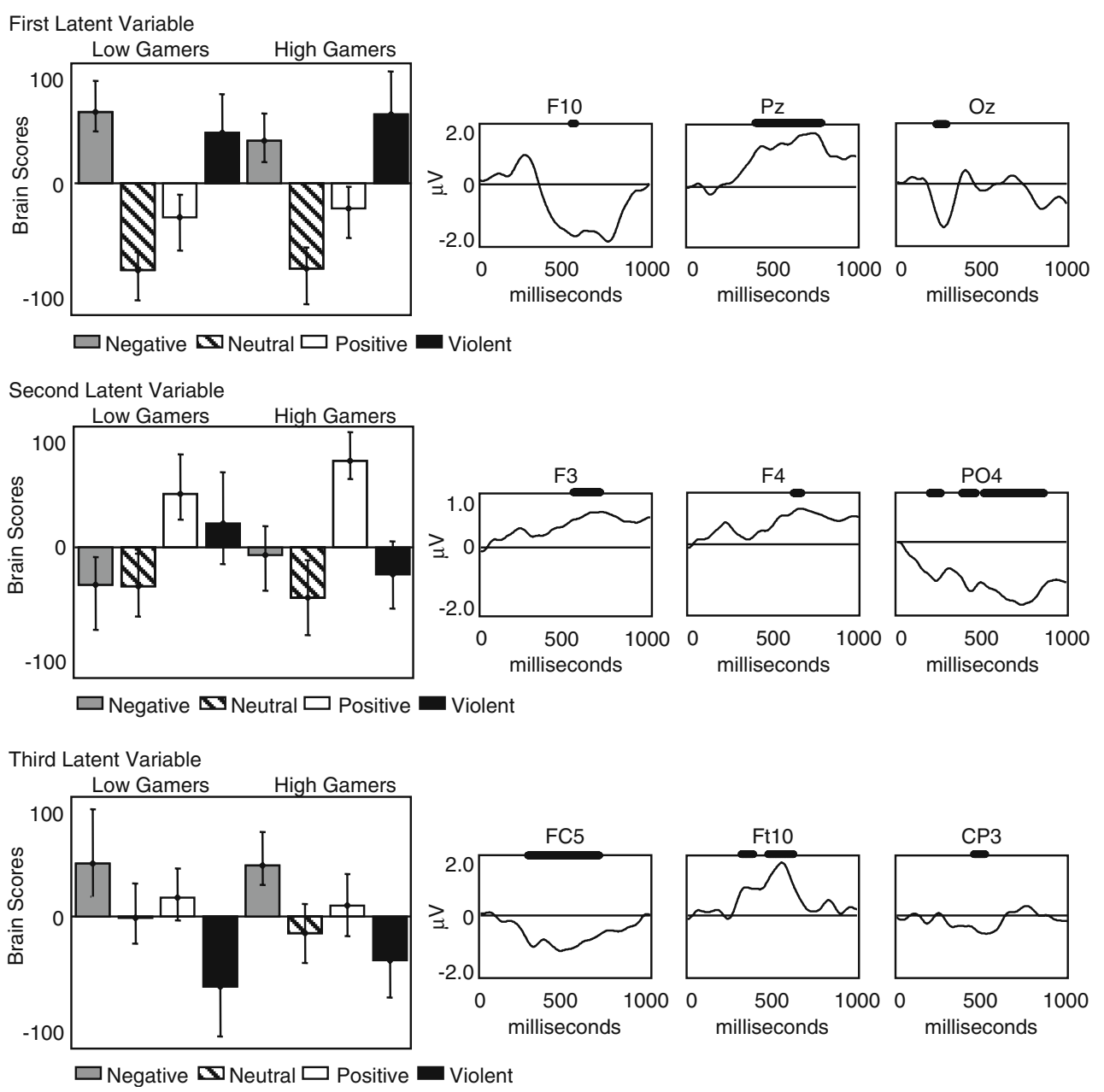

The second latent variable represented a contrast between positive pictures and neutral pictures (Fig. 6, bottom panel), appearing to represent the processing of positive information. The magnitude of the brain scores for positive pictures was similar in low and high gamers, indicating that VGV exposure was not associated with positive affect. The electrode saliences revealed a sustained modulation over the frontal-central and parietal regions over most of the analyzed epoch (electrodes Fpz, Fz, Oz).

Threat-rating task The permutation test revealed three significant latent variables $(p=.001, p=.001, p=.03)$ that accounted for $71.25 \%, 16.70 \%$, and $7.03 \%$ of the cross-block covariance, respectively. The first latent variable represented a contrast between negative and violent pictures and neutral and positive pictures (Fig. 7, top panel), appearing to capture the negativity bias. The magnitude of the brain scores was greater for violent pictures than for negative pictures, and this difference was similar for low and high gamers. This finding may indicate that directing attention to the level of threat represented in the pictures increases the salience of the violence portrayed in the pictures. The electrode saliences for the first latent variable revealed a sustained positivity over the parietal region and negativity over the lateral frontal regions between 400 and 1,200 ms after stimulus onset, reflecting the LPP (electrodes Pz, F9, F10).

In the low gamers, the second latent variable contrasted neutral and violent pictures with negative and positive pictures (Fig. 7, middle panel), and in the high gamers, this latent variable contrasted only neutral pictures with positive pictures. The electrode saliences revealed a single stable time period of neural activity that reflected a sustained negativity extending from the frontal to the central-parietal region (electrodes $\mathrm{Fz}, \mathrm{CPz}$ ) and a positivity over the occipital region between 400 and 1,200 ms after stimulus onset (electrode Iz).

The third latent variable reflected a contrast of negative and violent pictures in the high gamers (Fig. 7, bottom panel). In the low gamers, only the brain score for negative pictures was different from zero. The electrode saliences revealed a single time period of stable neural activity that reflected a sustained negativity over the left central region (electrode C5) and positivity that extended from the temporal to the lateral frontal region over the right hemisphere between 600 and $800 \mathrm{~ms}$ after stimulus onset 
Fig. 6 Results of the PLS analysis for the pleasantness rating task in Experiment 2. The top panel portrays the brain scores (left) and electrode saliences (right) for the first latent variable. The bottom panel portrays the brain scores and electrode saliences for the second latent variable. The error bars for the brain scores represent the $95 \%$ confidence intervals derived from the bootstrap distribution
First Latent Variable

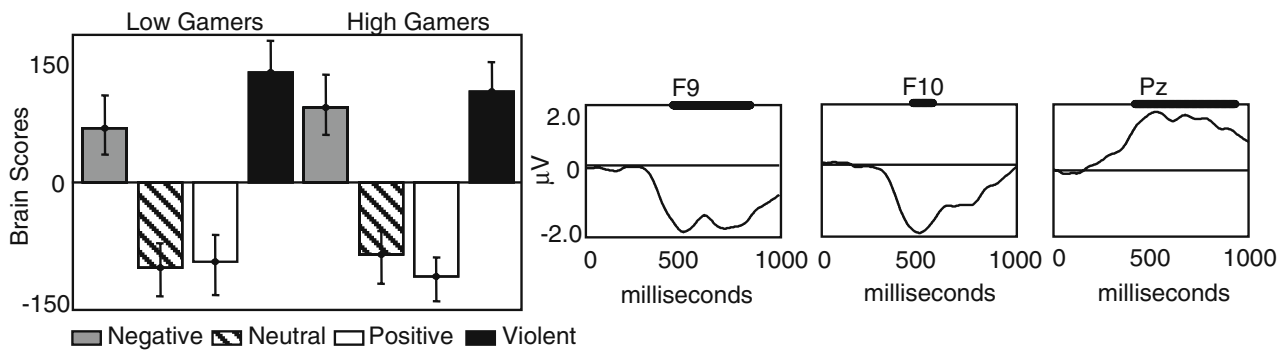

Second Latent Variable

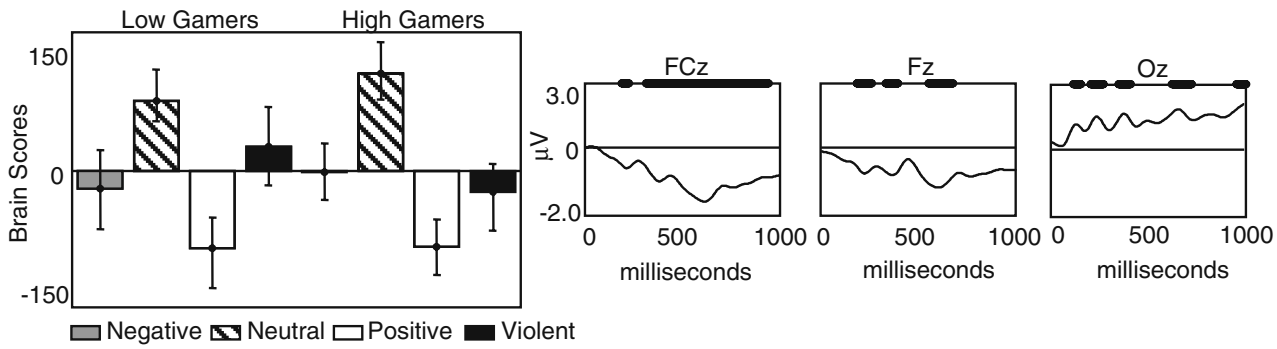

First Latent Variable
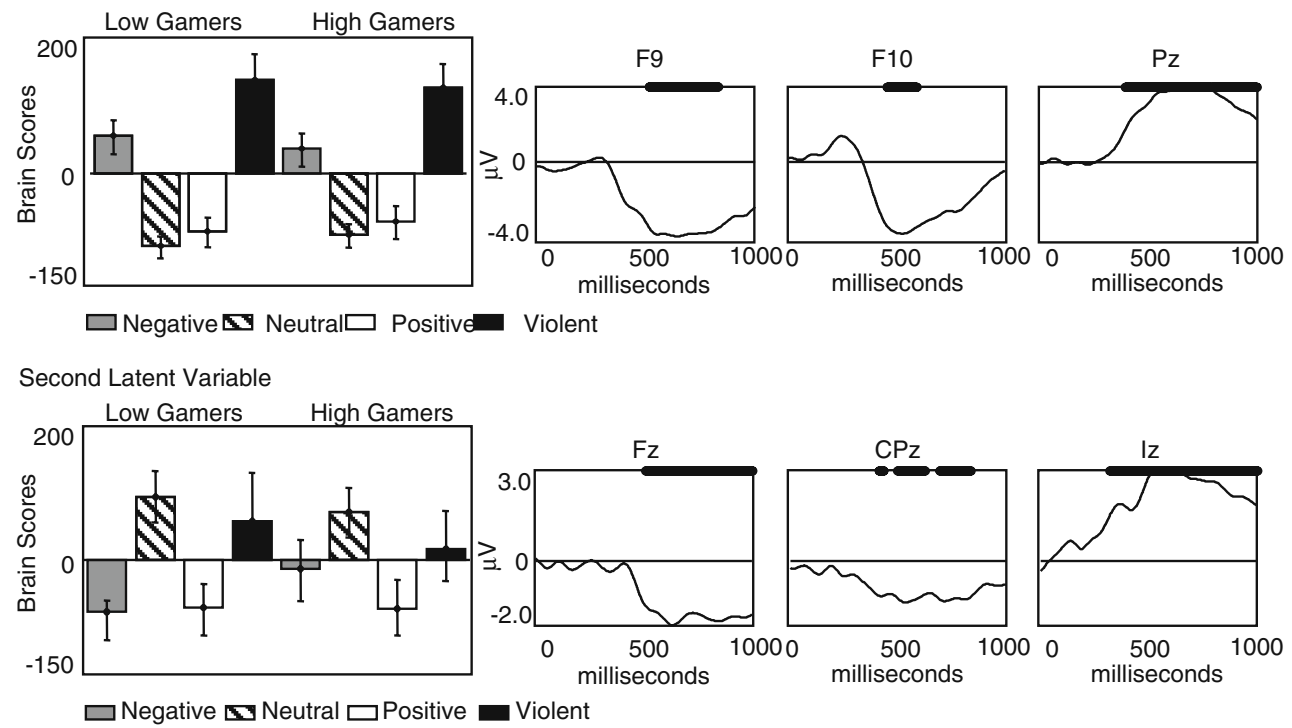

$\square$ Negative

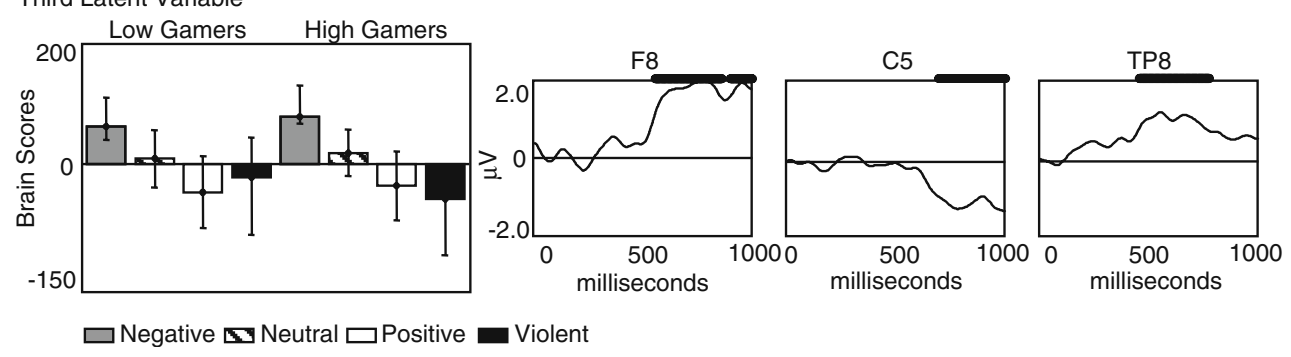

Fig. 7 Results of the PLS analysis for the threat-rating task in Experiment 2. The top panel portrays the brain scores (left) and electrode saliences (right) for the first latent variable. The middle panel portrays the brain scores and electrode saliences for the second latent variable. The bottom panel portrays the brain scores and electrode saliences for the third latent variable. The error bars for the brain scores represent the $95 \%$ confidence intervals derived from the bootstrap distribution 
(electrodes F8, TP8). The pattern of stable brain scores for this latent variable is somewhat consistent with the third latent variable for the colorfulness-rating task. Differences between the two analyses may indicate that the effect is attenuated, particularly in low gamers, when attention is directed to the level of threat represented in the stimuli.

\section{Discussion}

Experiment 2 reveals a number of interesting findings related to the association between chronic VGV exposure and the neural correlates of affective picture processing. For each task, the first latent variable appeared to capture the negativity bias, reflecting a contrast of negative and violent pictures with positive and neutral pictures. Focusing individuals on the level of threat represented in the pictures appears both to enhance the neural response to violent pictures and to eliminate differences between high and low gamers in the expression of the negativity bias that may exist for the other rating tasks. This finding may indicate that the association between VGV exposure and affective picture processing is moderated by the goals of the individuals, rather than reflecting an automatic response that influences affective information processing uniformly across task contexts.

The second latent variable appeared to reflect the neural correlates of processing positive emotion. For the pleasantness-rating task, the pattern of brain scores was similar for high and low gamers. In contrast, for the other tasks, the contribution of negative pictures in the low gamers varied with task demands. Within the context of work by Kirsh et al. (2006), the results of the present analyses fail to reveal a consistent association between VGV exposure and the neural correlates of processing positive affect.

In the colorfulness- and threat-rating tasks, the third latent variable generally reflected a contrast between negative and violent pictures. For the colorfulness-rating task this contrast appeared to be stronger for low gamers than for high gamers; in contrast, the opposite was true for the threat-rating task. Together, these findings may indicate that differences in the neural response to negative and violent pictures interact with the attentional orientation of the individual and individual differences in VGV.

\section{Combined analysis of color-rating task}

In Experiments 1 and 2, we observed a number of associations between chronic VGV exposure and the neural correlates of affective picture processing. One limitation of these results is that VGV exposure was treated as an extreme group variable, making it impossible to determine whether or not these associations are linear in nature. To further examine the nature of the association between VGV exposure and the neural correlates of affective picture processing, the ERP data for the color-rating task for the two experiments were combined. A median split was performed on the hours played per week for the high gamers, creating a group of 23 mid gamers $(M=16 \mathrm{~h}$ per week, $S D=5)$ and 22 high gamers $(M=42 \mathrm{~h}$ per week, $S D=14)$. A group of low gamers was formed by randomly selecting 23 or the 45 low gamers from across the two experiments $(M=3 \mathrm{~h}$ per week, $S D=4)$.

The grand-averaged ERPs at four midline electrodes are presented in Fig. 8. The permutation test for the PLS analysis revealed three significant latent variables $(p=.001$, $p=.001, p=.002)$ that accounted for $42.37 \%, 24.45 \%$, and $15.32 \%$ of the cross-block covariance, respectively. The first latent variable represented a contrast between negative and violent pictures and neutral and positive pictures (Fig. 9, top panel), appearing to capture the negativity bias. As in Experiment 1, the magnitude of the brain score for negative pictures was similar for the low and high gamers, while the size of the brain score for violent pictures was reduced in the mid and high gamers, relative to the low gamers. The electrode saliences revealed two stable time periods of neural activity. The first reflected a transient negativity over the occipital region around $280 \mathrm{~ms}$ after stimulus onset, likely reflecting the EPN (electrode Oz). The second reflected a sustained positivity over the central and parietal regions $500-1,000 \mathrm{~ms}$ after stimulus onset, likely reflecting the LPP (electrodes CP3, Pz).

The second latent variable represented a contrast between positive pictures and neutral pictures (Fig. 9, middle panel) and revealed a quadratic association with gaming status (i.e., it increased from low to mid gamers and then decreased from mid to high gamers). The electrode saliences revealed one stable time period of neural activity, reflecting a sustained negativity over the frontal-central region between 500 and $800 \mathrm{~ms}$ and a positivity over the parietal-occipital region between 400 and 1,000 ms (electrodes $\mathrm{FCz}, \mathrm{PO} 10, \mathrm{Oz}$ ).

The third latent variable represented a contrast between negative pictures and violent pictures (Fig. 9, bottom panel), with the strength of the contrast decreasing in a linear fashion from low gamers to high gamers. The electrode saliences revealed two stable time periods of neural activity. The first reflected a sustained negativity over the lateral frontal region and positivity over the central parietal region that persisted for most of the epoch (electrodes F9, TP8). The second reflected a transient positivity over the parietal-occipital region that peaked around $300 \mathrm{~ms}$ (electrode PO9). 
Fig. 8 Grand-averaged ERPs at four midline electrodes for the combined analysis of Experiments 1 and 2 demonstrating the time course of the EPN (white arrow) and LPP (black arrow) in low, mid, and high gamers in the color-rating task. The taller bar marks the onset of the stimulus, while the shorter bars represent 200-ms increments, and positive is plotted up
Low Gamers

Mid Gamers

High Gamers

Fpz
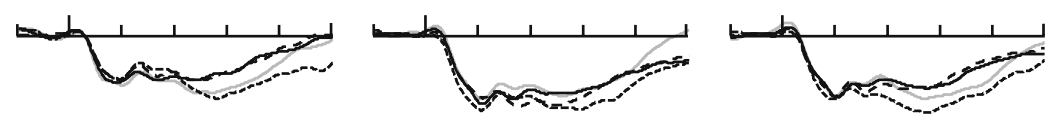

FCz
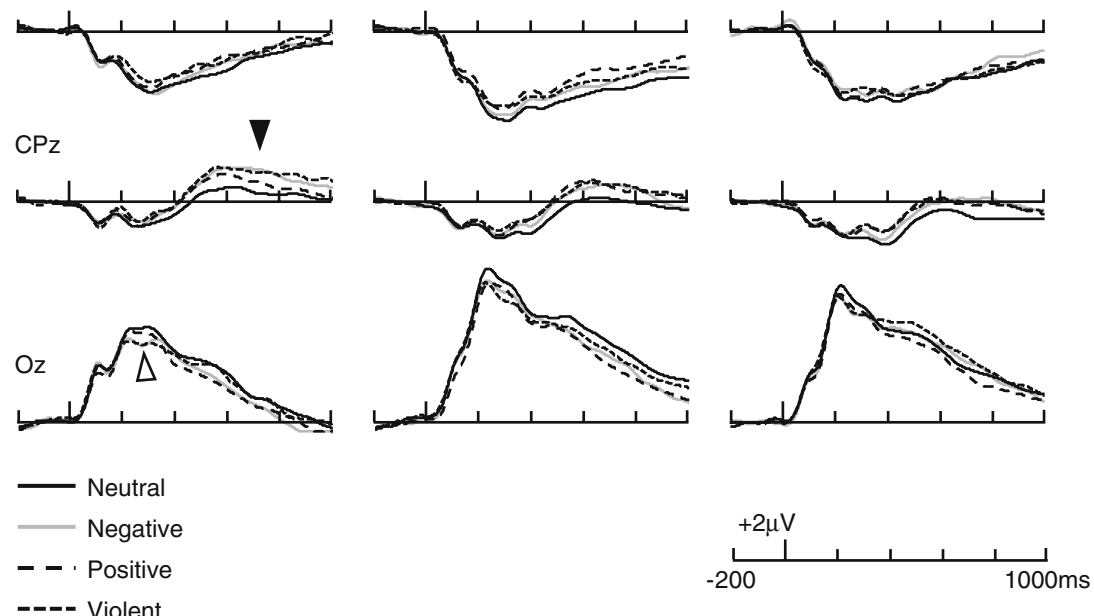

Following the findings of Bartholow et al. (2006), the first and third latent variables appear to indicate that VGV exposure is associated with changes in one's sensitivity to violent stimuli. The results of the combined analysis also indicate that there may be both linear and nonlinear associations between the level of chronic VGV exposure and the neural correlates of affective picture processing.

\section{General discussion}

The present study examined the association between chronic VGV exposure and affective picture processing. Past research indicates that VGV exposure is associated with desensitization to violent stimuli (Anderson et al., 2010; Bartholow et al., 2006) and changes in the processing of facial expressions (Kirsh et al., 2006). The present investigation was designed to extend previous work, examining the association between VGV exposure and the processing of positive emotion in stimuli other than faces, to determine whether desensitization to violence is observed with a single presentation of the stimuli and to ascertain whether the association between VGV exposure and desensitization to violent stimuli was sensitive to the relevance of emotion for task performance. The data extend previous research by demonstrating that VGV exposure is associated with differences in early transient processing associated with attentional orienting (i.e., transient frontal modulations and the EPN) and more sustained activity related to evaluative processing (i.e., LPP).
The negativity bias

The negativity bias was clearly seen in the first latent variable of each of the PLS analyses, reflecting a contrast of negative and violent pictures with neutral and positive pictures. This finding is consistent with previous research using ERPs to examine the neural correlates of the negativity bias (Ito \& Cacioppo, 2005; Ito et al., 1998) and recent work from our laboratory using PLS analysis to identify the ERP correlates of the negativity bias (Bailey et al., 2011). Two aspects of the data lead to the suggestion that the negativity bias represents an effect of valence rather than of arousal. First, normative arousal ratings overlapped for the positive and negative pictures; second, there were no systematic differences in the magnitude of the brain scores for positive and neutral picture across the analyses, even though these stimuli differed in arousal. Across the two experiments, three distinct components of the ERPs were sensitive to the negativity bias. The earliest expression of the negativity bias reflected a transient modulation of the ERPs over the frontal region between 100 and $200 \mathrm{~ms}$ after stimulus onset. The next manifestation of the negativity bias was seen as a modulation of the amplitude of the EPN over the parietal-occipital region between roughly 200 and $300 \mathrm{~ms}$ after stimulus onset. Finally, the negativity bias modulated the amplitude of the LPP over the parietal region between 400 and 1,000 ms after stimulus onset. These data are consistent with recent work by Foti et al. (2009) demonstrating that multiple components of the ERPs are sensitive to the affective information represented in pic- 
Fig. 9 Results of the PLS analysis for the combined data for the color-rating tasks from Experiments 1 and 2. The top panel portrays the brain scores (left) and electrode saliences (right) for the first latent variable. The middle panel portrays the brain scores and electrode saliences for the second latent variable. The bottom panel portrays the brain scores and electrode saliences for the third latent variable. The error bars for the brain scores represent the $95 \%$ confidence intervals derived from the bootstrap distribution

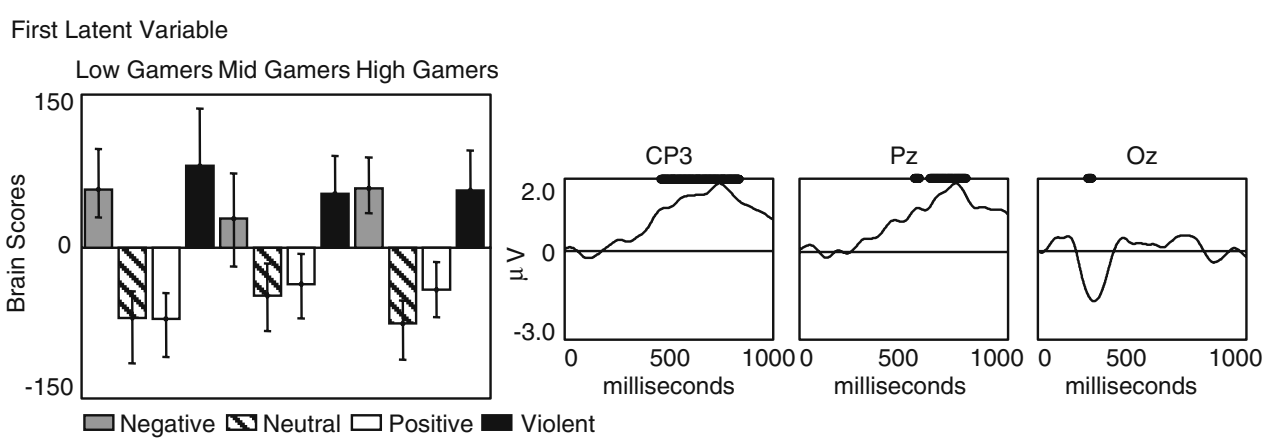

Second Latent Variable

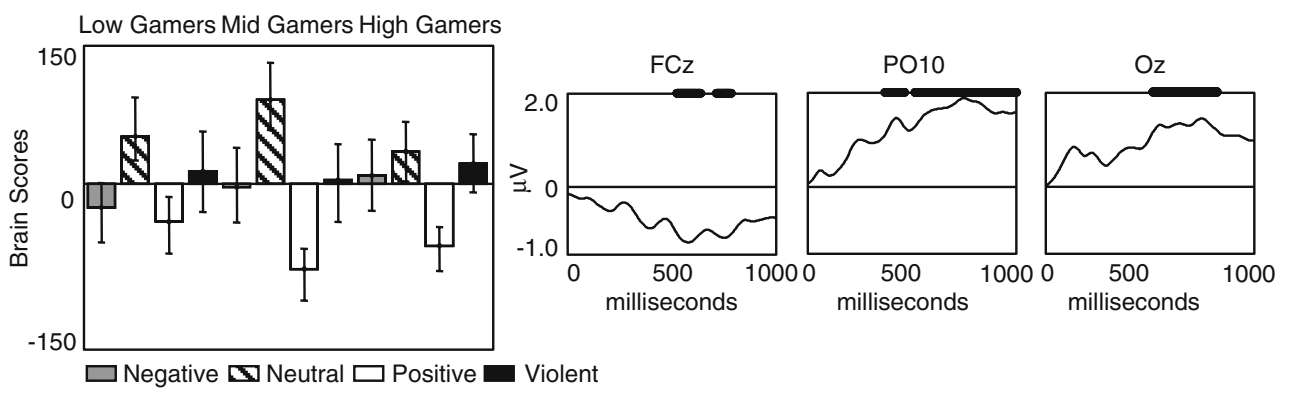

Third Latent Variable

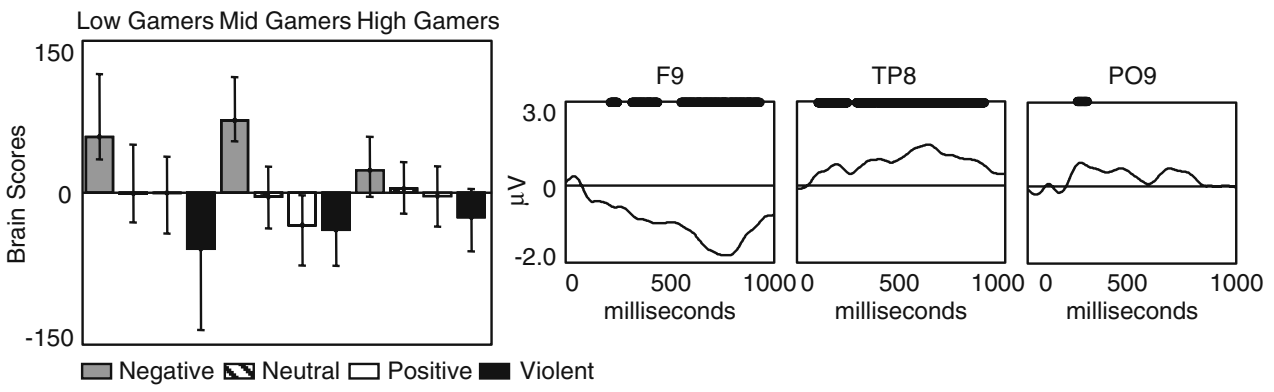

tures, with some of these components being differentially sensitive to positive and negative valence.

The presence of the negativity bias in the present experiments is inconsistent with recent work demonstrating that this effect may represent an artifact of the nature of the stimuli that are included in the design (Weinberg \& Hajcak, 2010). Specifically, these investigators found that the negativity bias was eliminated when erotic stimuli were included in the comparison of negative and positive pictures and that the effect was observed when negative pictures were compared with positive nonerotic pictures. Recent work from our laboratory replicated this pattern of data when measures of mean voltage were used to quantify differences in neural activity between positive and negative pictures (Bailey et al., 2011). However, when PLS analysis was applied to the same dataset,a latent variable was observed that contrasted the violent and negative pictures used in the present experiments with the positive pictures used in the present experiments and a set of erotic pictures. Given these findings, it seems that the negativity bias does represent a robust attribute of affective information pro- cessing (Baumeister et al., 2001). However, it also appears that further research is required in order to gain a clearer understanding of the impact of variation in stimulus attributes on this effect.

Differences in the expression of the negativity bias in the color-rating task, where emotion was not relevant to task performance, and in the pleasantness- and threat-rating tasks, where emotion was relevant to task performance, reveals that this effect is sensitive to the attentional demands of the orienting task, rather than reflecting a relatively automatic effect (Ito \& Cacioppo, 2005; Ito et al., 1998; Smith, Cacioppo, Larsen, \& Chartrand, 2003). Specifically, when emotion was not relevant to task performance in the colorfulness-rating task, the magnitude of the brain scores for negative and violent pictures was relatively similar in the PLS analysis. In contrast, the magnitude of the brain scores was greater for violent pictures than for negative pictures when emotion (i.e., pleasantness or level of threat) was relevant to task performance. On the basis of these findings, it appears that making an affective judgment about the pictures, regardless 
of the valence of the judgment, served to potentiate the negativity bias for violent pictures. The present findings lead to the suggestion that the relative importance of various types of negative information-for instance, violence versus grief and loss-may be influenced by the context in which the stimuli are presented (Baumeister et al., 2001).

\section{ERPs and positive emotion}

The second latent variable from the PLS analyses appeared to capture the ERP correlates of processing positive emotion. In each of the analyses, this latent variable contrasted positive pictures and neutral pictures. This finding is consistent with some previous research revealing differential neural activity for positive stimuli relative to neutral stimuli (Carretie et al., 2006; Codispoti et al., 2007; Weinberg \& Hajcak, 2010). The contribution of negative and violent pictures to the second latent variable varied across the experimentsand rating tasks and with gamer status (e.g., for Experiment 1, the second latent variable contrasted neutral pictures with positive and violent pictures in the high gamers, while in Experiment 2, the second latent variable contrasted positive pictures with neutral and negative pictures in the low gamers). It is not clear what factors contributed to variation in the expression of the second latent variable in the different analyses, leading to the suggestion that this is one avenue for future investigation.

If one focuses on the brain scores for positive and neutral pictures in the second latent variable, there appears to be relatively little association between chronic VGV exposure and the neural correlates of processing positive affective information, with the possible exception of the quadratic association observed in the combined analysis of the colorfulness-rating data. The present data reveal that the negative association between VGV and processing positive affective information observed by Kirsh and colleagues (Kirsh et al., 2006; Kirsh \& Mounts, 2007) may be limited to emotion carried in the face.

\section{VGV exposure and desensitization to violence}

Consistent with Bartholow et al. (2006), the present study showed some evidence for desensitization to violent pictures in high gamers, relative to low gamers. There were two latent variables that appeared to express the association between VGV exposure and desensitization to violence. For the first latent variable in Experiment 1, the magnitude of the brain scores for negative pictures was similar for low and high gamers, while the magnitude of the brain scores for violent pictures was reduced in high gamers, relative to low gamers. This general pattern was also observed for the first latent variable of the combined analysis and appeared to reflect differences between low, and mid and high gamers. The interaction between gaming status and the processing of negative and violent pictures was reduced or eliminated when emotion was relevant to task performance in the pleasantness- and threat-rating tasks, leading to the suggestion that the expression of desensitization on the negativity bias was sensitive to the attentional or processing demands of the task.

The pattern of brain scores for the third latent variable in the analysis for the colorfulness- and threat-rating tasks for Experiment 2 and the combined analysis also provided some evidence for desensitization to violence in high gamers. For the color-rating task and in the combined analysis, the magnitude of the difference between negative and violent pictures decreased as VGV exposure increased, as might be expected if VGV exposure is associated with desensitization to violence. In contrast, for the threat-rating task, the contrast between negative and violent pictures was stronger for high gamers than for low gamers. This finding, together with the lack of an association between VGV exposure and affective picture processing for the first latent variable in the threat-rating task, may again indicate that the expression of desensitization to violence in high gamers is dependent on the processing demands of the orienting task that is being performed.

\section{Limitations and conclusions}

A limitation of the present study is that VGV exposure was measured as an individual-difference variable, rather than being induced in an experimental study. This makes it possible that group differences other than VGV exposure per se may drive the association between gamer status and affective picture processing. One plausible alternative would be that people who are already desensitized to violence choose to spend more time playing violent video games. While we cannot address this alternative in our data, there is a large body of evidence demonstrating that increased levels of aggression and desensitization to violence are consistently observed in studies using a variety of methods, including those involving individual differences (Bartholow et al., 2006), those examining the effect of acute VGV exposure (Carnagey et al., 2007), and those assessing longitudinal changes in aggression associated with VGV exposure after controlling for baseline levels of trait aggression and VGV exposure (Anderson et al., 2010). Given the robustness of the association between VGV exposure and desensitization to violence, it seems reasonable to suggest that at least some of the association between gamer status and the neural correlates of affective picture processing observed in the present study resulted from VGV exposure. 
A second alternative may be that individuals with certain personality traits are more susceptible to the effects of VGV exposure. For example, anger and psychoticism have been found to moderate the effect of VGV exposure on aggression, such that individuals who are high on these traits display greater aggression after VGV exposure than do individuals who are low on these traits (Giumetti \& Markey, 2007; Markey \& Scherer, 2009; Peng, Liu, \& Mou, 2008). In the present study, low and high gamers reported similar levels of anger (Experiment 2); therefore, it seems unlikely that individual differences in this variable account for the relationship between gamer status and affective picture processing. In a recent study, Markey and Markey (2010) demonstrated that the individuals most susceptible to the effects of VGV exposure on aggression were high on neuroticism and low on conscientiousness and agreeableness. This cluster of personality variables was not measured in the present study, so considering the potential influence of these personality characteristics represents one avenue of future research.

In conclusion, the present study demonstrates that VGV exposure is associated with variation in the neural correlates of affective picture processing. Consistent with the findings of Bartholow et al. (2006), desensitization to violent pictures was observed in high gamers, relative to low gamers, in some task conditions. The nature of the association between VGV exposure and the neural correlates of affective picture processing reflected both linear and nonlinear effects, meaning that it may be important to consider continuous in addition to extreme group samples in future studies. The association between VGV exposure and affective picture processing was moderated by the orienting task being performed, suggesting that care should be taken when interpreting the results of a single comparison within this area of research. In contrast to previous research using faces, there appears to be little association between VGV exposure and the neural correlates of processing positive affective information.

\section{References}

Anderson, C. A., \& Bushman, B. J. (2002). Human aggression. Annual Review of Psychology, 53, 27-51.

Anderson, C. A., Gentile, D. A., \& Buckley, K. E. (2007). Violent video game effects on children and adolescents: Theory, research, and public policy. Oxford: Oxford University Press.

Anderson, C. A., Shibuya, A., Ihori, N., Swing, E. L., Bushman, B. J., Sakamoto, A., et al. (2010). Violent video game effects on aggression, empathy, and prosocial behavior in Eastern and Western countries. Psychological Bulletin, 136, 151-173.

Bailey, K., West, R., Olson, K. (2011). Distinct neural correlates of the negativity bias and processing sexually arousing pictures. Manuscript submitted for publication

Barlett, C. P., Anderson, C. A., \& Swing, E. L. (2009). Video game effects confirmed, suspected and speculative: A review of the evidence. Simulation \& Gaming, 40, 377-403.
Bartholow, B. D., Bushman, B. J., \& Sestir, M. A. (2006). Chronic violent video game exposure and desensitization to violence: Behavioral and event-related brain potential data. Journal of Experimental Social Psychology, 42, 532-539.

Baumeister, R. F., Bratslavsky, E., Finkenauer, C., \& Vohs, K. D. (2001). Bad is stronger than good. Review of General Psychology, 5, 323-370.

Beck, A. T., Ward, C. H., Mendelson, M., Mock, J., \& Erbaugh, J. (1961). An inventory for measuring depression. Archives of General Psychiatry, 4, 561-571.

Billings, L. S., Harrison, D. W., \& Alden, J. D. (1993). Age differences among women in the functional asymmetry for bias in facial affect perception. Bulletin of the Psychonomic Society, 31, 317-320.

Bushman, B. J., \& Anderson, C. A. (2009). Comfortably numb: Desensitizing effects of violent media on helping others. Psychological Science, 20, 273-277.

Carnagey, N. L., Anderson, C. A., \& Bushman, B. J. (2007). The effect of video game violence on physiological desensitization to real-life violence. Journal of Experimental Social Psychology, 43, 489-496.

Carretie, L., Hinojosa, J. A., Albert, J., \& Mercado, F. (2006). Neural response to sustained affective visual stimulation using an indirect task. Experimental Brain Research, 174, 630-637.

Carver, C. S., \& White, T. L. (1994). Behavioral inhibition, behavioral activation, and affective responses to impending reward and punishment: The BIS/BAS scales. Journal of Personality and Social Psychology, 67, 319-333.

Codispoti, M., Ferrari, V., \& Bradley, M. M. (2007). Repetition and event-related potentials: Distinguishing early and late processes in affective picture perception. Journal of Cognitive Neuroscience, 19, 577-586.

Entertainment Software Association. (2010). Retrieved July 12, 2010, from $\mathrm{http}: / / \mathrm{www}$.theesa.com/facts/index.asp.

Foti, D., Hajcak, G., \& Dien, J. (2009). Differentiating neural responses to emotional pictures: Evidence from temporal-spatial PCA. Psychophysiology, 46, 521-530.

Funk, J. B., Baldacci, H. B., Pasold, T., \& Baumgardner, J. (2004). Violence exposure in real-life, video games, television, movies, and the Internet: Is there desensitization? Journal of Adolescence, 27, 23-39.

Gentile, D. (2009). Pathological video-game use among youth ages 8 to 18. Psychological Science, 20, 594-602.

Gentile, D. A., Anderson, C. A., Yukawa, S., Ihori, N., Saleem, M., Ming, L. K., et al. (2009). The effects of prosocial video games on prosocial behaviors: International evidence from correlational, experimental, and longitudinal studies. Personality and Social Psychology Bulletin, 35, 752-763.

Giumetti, G. W., \& Markey, P. M. (2007). Violent video games and anger as predictors of aggression. Journal of Research in Personality, 41, 1234-1243.

Gorsuch, R. L. (1983). Factor analysis (2nd ed.). Hillsdale, NJ: Erlbaum.

Hajcak, G., Dunning, J. P., \& Foti, D. (2009). Motivated and controlled attention to emotion: Time-course of the late positive potential. Clinical Neurophysiology, 120, 505-510.

Ito, T. A., \& Cacioppo, J. T. (2005). Variations on a human universal: Individual differences in positivity offset and negativity bias. Cognition and Emotion, 19, 1-26.

Ito, T. A., Larsen, J. T., Smith, N. K., \& Cacioppo, J. T. (1998). Negative information weighs more heavily on the brain: The negativity bias in evaluative categorizations. Journal of Personality and Social Psychology, 75, 887-900.

Keil, A., Bradley, M. M., Hauk, O., Rockstroh, B., Elbert, T., \& Lang, P. J. (2002). Large-scale neural correlates of affective picture processing. Psychophysiology, 39, 641-649.

Kirsh, S. J., \& Mounts, J. R. W. (2007). Violent video game play impacts facial emotion recognition. Aggressive Behavior, 33, 353-358. 
Kirsh, S. J., Mounts, J. R. W., \& Olczak, P. V. (2006). Violent media consumption and the recognition of dynamic facial expressions. Journal of Interpersonal Violence, 21, 571-584.

Lang, P. J., Bradley, M. M., \& Cuthbert, B. N. (2005). International affective picture system (IAPS): Technical manual and affective ratings. Gainesville: University of Florida, Center for Research in Psychophysiology.

Leppanen, J., Tenhunen, M., \& Hietanen, J. (2003). Faster choicereaction times to positive than to negative facial expressions. Journal of Psychophysiology, 17, 113-123.

Lobaugh, N. J., West, R., \& McIntosh, A. R. (2001). Spatiotemporal analysis of experimental differences in event-related potential data with partial least squares. Psychophysiology, 38, 517-530.

Markey, P. M., \& Markey, C. N. (2010). Vulnerability to violent video games: A review and integration of personality research. Review of General Psychology, 14, 82-91.

Markey, P. M., \& Scherer, K. (2009). An examination of psychoticism and motion capture controls as moderators of the effects of violent video games. Computers in Human Behavior, 25, 407-411.

McIntosh, A. R., \& Lobaugh, N. J. (2004). Partial least squares analysis of neuroimaging data: Applications and advances. Neuroimage, 23, S250-S263.

Oldfield, R. C. (1971). The assessment and analysis of handedness: The Edinburgh Inventory. Neuropsychologia, 9, 97-113.
Olofsson, J. K., Nordin, S., Sequeira, H., \& Polich, J. (2008). Affective picture processing: An integrative review of ERP findings. Biological Psychology, 77, 247-265.

Peng, W., Liu, M., \& Mou, Y. (2008). Do aggressive people play violent computer games in a more aggressive way? Individual difference and idiosyncratic game-playing experience. Cyberpsychology \& Behavior, 11, 157-161.

Schupp, H. T., Cuthbert, B. N., Bradley, M. M., Cacioppo, J. T., Ito, T., \& Lang, P. J. (2000). Affective picture processing: The late positive potential is modulated by motivational relevance. Psychophysiology, 37, 257-261.

Schupp, H. T., Junghofer, M., Weike, A. I., \& Hamm, A. O. (2003). Emotional facilitation of sensory processing in the visual cortex. Psychological Science, 14, 7-13.

Schupp, H. T., Junghofer, M., Weike, A. I., \& Hamm, A. O. (2004). The selective processing of briefly presented affective pictures: An ERP analysis. Psychophysiology, 41, 441-449.

Smith, N. K., Cacioppo, J. T., Larsen, J. T., \& Chartrand, T. L. (2003). May I have your attention, please: Electrocortical responses to positive and negative stimuli. Neuropsychologia, 41, $171-183$.

Weinberg, A., \& Hajcak, G. (2010). Beyond good and evil: The timecourse of neural activity elicited by specific picture content. Emotion, 10, 767-782. 